\title{
Estimation in Step-Stress Accelerated Life Tests for Power Generalized Weibull Distribution with Progressive Censoring
}

\author{
M. M. Mohie EL-Din, ${ }^{1}$ S. E. Abu-Youssef, ${ }^{1}$ Nahed S. A. Ali, ${ }^{2}$ and A. M. Abd El-Raheem ${ }^{2}$ \\ ${ }^{1}$ Department of Mathematics, Faculty of Science, Al-Azhar University, Cairo, Egypt \\ ${ }^{2}$ Department of Mathematics, Faculty of Education, Ain Shams University, Cairo, Egypt
}

Correspondence should be addressed to A. M. Abd El-Raheem; a_m2am@yahoo.com

Received 22 October 2014; Accepted 20 January 2015

Academic Editor: Shuo-Jye Wu

Copyright (C) 2015 M. M. Mohie EL-Din et al. This is an open access article distributed under the Creative Commons Attribution License, which permits unrestricted use, distribution, and reproduction in any medium, provided the original work is properly cited.

Based on progressive censoring, step-stress partially accelerated life tests are considered when the lifetime of a product follows power generalized Weibull distribution. The maximum likelihood estimates (MLEs) and Bayes estimates (BEs) are obtained for the distribution parameters and the acceleration factor. In addition, the approximate and bootstrap confidence intervals (CIs) of the estimators are presented. Furthermore, the optimal stress change time for the step-stress partially accelerated life test is determined by minimizing the asymptotic variance of MLEs of the model parameters and the acceleration factor. Simulation results are carried out to study the precision of the MLEs and BEs for the parameters involved.

\section{Introduction}

In reliability analysis, it is not easy to collect lifetimes on highly reliable products with very long lifetimes, because very few or even no failures may occur within a limited testing time under normal conditions. For this reason, accelerated life tests (ALTs) or partially accelerated life tests (PALTs) are one of the most common approaches that are used to obtain enough failure data, in a short period of time. In ALTs all test units are subjected to higher than usual levels of stress, to induce early failures. In PALTs units are tested at both accelerated and use conditions. The information obtained from the test performed in the accelerated or partially accelerated test is used to estimate the failure behavior of the units under normal conditions. The stress loading in ALTs can be applied in different ways. Commonly used methods are constant-stress and step-stress. Nelson [1] discussed the advantages and disadvantages of each of such methods.

In constant-stress ALT, each unit is run at constant high stress until either failure occurs or the test is terminated. In step-stress ALT, the stress on each unit is not constant but is increased step by step at prespecified times or upon the occurrence of a fixed number of failures. When a test involves two levels of stress with the first level as the normal condition and has a fixed time point for changing stress referred to as a step-stress partially ALT (SSPALT).

PALTs were studied under step-stress scheme by several authors; for example, see Goel [2], DeGroot and Goel [3], Bhattacharyya and Soejoeti [4], Bai and Chung [5], Ismail and Aly [6], and Abdel-Ghani [7].

In ALTs or PALTs, tests are often stopped before all units fail. The estimate from the censored data is less accurate than the estimate from complete data. However, censored data is more than offset by the reduced test time and expense. The most common censoring scheme is type-II censoring. Consider $n$ units are placed on life test, and the experimenter terminates the experiment after a prespecified number of units $m \leq n$ fail. In this scenario, only the smallest lifetimes are observed. In conventional type-II censoring schemes do not allow to remove units at points other than the terminal point of the experiment. A generalization of type-II censoring is the progressive type-II censoring. It is a method which enables an efficient exploitation of the available resources by continual removal of a prespecified number of surviving test units at each failure time. On other hand, the removal of units before failure may be intentional to save time and cost or when some items have to be removed for use in another experiment. 
This paper will concentrate on SSPALTs under progressive type-II censoring. It can be described as follows. Consider an experiment in which $n$ units are placed on a life testing experiment. At the time of the first failure, $R_{1}$ units are randomly removed from the remaining $n-1$ surviving units. Similarly, at the time of the second failure, $R_{2}$ units from the remaining $n-2-R_{1}$ units are randomly removed. The test continues until the $m$ th failure occurs at which time, all the remaining, $R_{m}=n-m-R_{1}-R_{2}-\cdots-R_{m-1}$ units are removed. If $R_{1}=R_{2}=\cdots=R_{m}=0$, then $n=m$, which is the complete sample situation. If $R_{1}=R_{2}=\cdots=R_{m-1}=0$, then $R_{m}=$ $n-m$ which corresponds to conventional type-II censoring. A recent account on progressive censoring schemes can be found in the book by Balakrishnan and Aggarwala [8].

The paper is organized as follows. In Section 2, a description of the model, test procedure, and its assumptions are presented. In Section 3, the MLEs of the SSPALT model parameters are derived. The BEs of model parameters using Lindley's approximation and MCMC method are obtained in Section 4. In Section 5, the approximate and bootstrap confidence bounds for the model parameters are constructed. In Section 6, estimation of optimal stress change time is obtained. Section 7 contains the simulation results. Conclusion is made in Section 8.

\section{Model Description}

2.1. Power Generalized Weibull Distribution. The power generalized Weibull (PGW) distribution is an extension of Weibull distribution. It was introduced by Bagdonavičius and Nikulin [9] as a baseline distribution for the accelerated failure time model. It not only contains distributions with unimodal and bathtub hazard shape but also allows for a broader class of monotone hazard rate.

The $\operatorname{PGW}(\gamma, \nu, \sigma)$ distribution is specified by the probability density function (pdf):

$$
\begin{array}{r}
f(t)=\frac{\gamma \nu}{\sigma^{\nu}} t^{\nu-1}\left(1+\left(\frac{t}{\sigma}\right)^{\nu}\right)^{(\gamma-1)} \exp \left\{1-\left(1+\left(\frac{t}{\sigma}\right)^{\nu}\right)^{\gamma}\right\}, \\
t>0, \quad \gamma>0, \quad \nu>0, \quad \sigma>0,
\end{array}
$$

the corresponding survival function is

$$
\begin{aligned}
& S(t)=\exp \left\{1-\left(1+\left(\frac{t}{\sigma}\right)^{\gamma}\right)^{\gamma}\right\}, \\
& t>0, \quad \gamma>0, \quad \nu>0, \quad \sigma>0,
\end{aligned}
$$

and the corresponding hazard rate function is given by

$$
h(t)=\frac{\gamma \nu}{\sigma^{\nu}} t^{\nu-1}\left(1+\left(\frac{t}{\sigma}\right)^{\nu}\right)^{\gamma-1} .
$$

Particular cases of the power generalized Weibull distribution are as follows.

(1) If $\gamma=1$, PGW distribution tends to Weibull distribution.

(2) If $\gamma=1$ and $\nu=1$, PGW distribution tends to exponential distribution.
2.2. Assumptions and Test Procedure. The following assumptions are used throughout the paper in the framework of SSPALT.

(1) $n$ identical and independent items are put on a life test.

(2) The lifetime of each unit has $\operatorname{PGW}(\gamma, \nu, 1)$ distribution.

(3) The test is terminated at the time of the $m$ th failure, where $m$ is prefixed $(m \leq n)$.

(4) Each of the $n$ items is run under normal use condition. If it does not fail or remove from the test by a prespecified time $\tau$, it is put under accelerated condition.

(5) At the time of the $i$ th failure, a random number of the surviving items $R_{i}, i=1,2, \ldots, m-1$, are randomly selected and removed from the test. Finally, at the time of the $m$ th failure, the remaining surviving items $R_{m}=n-m-\sum_{i=1}^{m-1} R_{i}$ are removed from the test and the test is terminated.

(6) Let $n_{1}$ be the number of failures before time $\tau$ at normal condition, and let $m-n_{1}$ be the number of failures after time $\tau$ at stress condition, then, the observed progressive censored data are

$y_{1 ; m, n}^{R}<\cdots<y_{n_{1} ; m, n}^{R}<\tau<y_{n_{1}+1 ; m, n}^{R}<\cdots<y_{m ; m, n}^{R}$,

where $R=\left(R_{1}, R_{2}, \ldots, R_{m}\right)$ and $\sum_{i=1}^{m} R_{i}=n-m$.

(7) The tampered random variable (TRV) model holds. It was proposed by DeGroot and Goel [3]. According to tampered random variable model the lifetime of a unit under SSPALT can be written as

$$
Y= \begin{cases}T, & \text { if } T \leq \tau, \\ \tau+\frac{1}{\beta}(T-\tau), & \text { if } T>\tau,\end{cases}
$$

where $T$ is the lifetime of the units under normal condition, $\tau$ is the stress change time, and $\beta$ is the acceleration factor $(\beta>1)$.

(8) From the TRV model in (5), the pdf of two parameters $\operatorname{PGW}(\gamma, \nu, 1)$ distribution under SSPALT is given by

$$
\begin{aligned}
& f(y)
\end{aligned}
$$

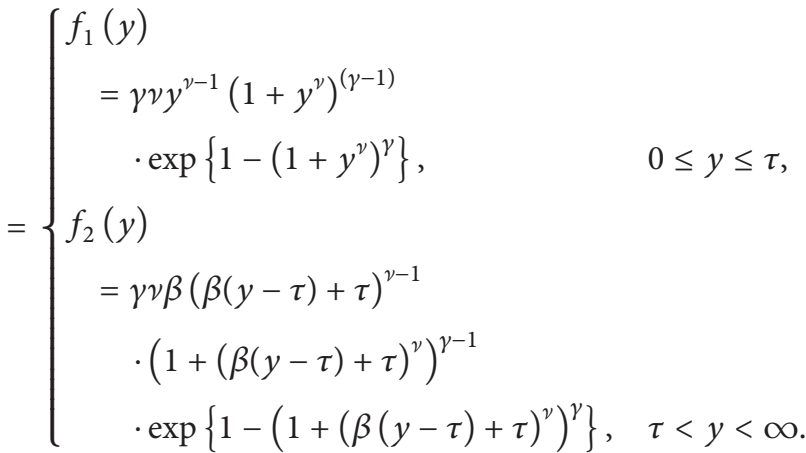




\section{Maximum Likelihood Estimation}

In this section, the MLEs of the model parameters are obtained. Let $y_{i}=y_{i ; m, n}^{R}, i=1,2, \ldots, m$, be the observed values of the lifetime $Y$ obtained from a progressive censoring scheme under SSPALT, with censored scheme $R=$ $\left(R_{1}, R_{2}, \ldots, R_{m}\right)$. The maximum likelihood function of the observations $y_{1}<\cdots<y_{n_{1}}<\tau<y_{n_{1}+1}<\cdots<y_{m}$ takes the following form:

$$
\begin{aligned}
L(\gamma, \nu, \beta)= & C\left\{\prod_{i=1}^{n_{1}} f_{1}\left(y_{i}\right)\left[1-F_{1}\left(y_{i}\right)\right]^{R_{i}}\right\} \\
& \cdot\left\{\prod_{i=n_{1}+1}^{m} f_{2}\left(y_{i}\right)\left[1-F_{2}\left(y_{i}\right)\right]^{R_{i}}\right\},
\end{aligned}
$$

where

$$
\begin{aligned}
C= & n\left(n-1-R_{1}\right) \\
& \cdot\left(n-2-R_{1}-R_{2}\right) \cdots\left(n-m+1-\sum_{i=1}^{m-1} R_{i}\right) .
\end{aligned}
$$

From (6) in (7), we get

$$
\begin{aligned}
& L(\gamma, \nu, \beta) \\
& =C\left\{\prod_{i=1}^{n_{1}} \gamma \nu y_{i}^{\nu-1}\left(1+y_{i}^{\nu}\right)^{\gamma-1}\right. \\
& \left.\cdot \exp \left\{\left(R_{i}+1\right)\left(1-\left(1+y_{i}^{\nu}\right)^{\gamma}\right)\right\}\right\} \\
& \cdot\left\{\prod_{i=n_{1}+1}^{m} \gamma \nu \beta\left(\psi_{i}(\beta)\right)^{\nu-1}\left(1+\left(\psi_{i}(\beta)\right)^{\nu}\right)^{\gamma-1}\right. \\
& \left.\cdot \exp \left\{\left(R_{i}+1\right)\left(1-\left(1+\left(\psi_{i}(\beta)\right)^{\nu}\right)^{\gamma}\right)\right\}\right\},
\end{aligned}
$$

where $\psi_{i}(\beta)=\tau+\beta\left(y_{i}-\tau\right)$.

The $\log$-likelihood function may then be written as

$$
\begin{aligned}
\ell(\gamma, \nu, \beta)= & \log C+m \log \gamma \nu+\left(m-n_{1}\right) \log \beta+(\nu-1) \\
& \cdot\left(\sum_{i=1}^{n_{1}} \log y_{i}+\sum_{i=n_{1}+1}^{m} \log \psi_{i}(\beta)\right)+(\gamma-1) \\
& \cdot\left(\sum_{i=1}^{n_{1}} \log \left[1+y_{i}^{\nu}\right]+\sum_{i=n_{1}+1}^{m} \log \left[1+\left(\psi_{i}(\beta)\right)^{\nu}\right]\right) \\
& +\sum_{i=1}^{n_{1}}\left(R_{i}+1\right)\left(1-\left(1+y_{i}^{\nu}\right)^{\gamma}\right) \\
& +\sum_{i=n_{1}+1}^{m}\left(R_{i}+1\right)\left(1-\left(1+\left(\psi_{i}(\beta)\right)^{\nu}\right)^{\gamma}\right),
\end{aligned}
$$

and thus we have the likelihood equations for $\gamma, \nu$, and $\beta$, respectively, as

$$
\begin{aligned}
& \frac{\partial \ell(\gamma, \nu, \beta)}{\partial \gamma}=\frac{m}{\gamma}+\sum_{i=1}^{n_{1}} \log \left(1+y_{i}^{\nu}\right) \\
& -\sum_{i=1}^{n_{1}}\left(R_{i}+1\right)\left(1+y_{i}^{\nu}\right)^{\gamma} \log \left(y_{i}^{\nu}+1\right) \\
& +\sum_{i=n_{1}+1}^{m} \log \left(1+\left(\psi_{i}(\beta)\right)^{\nu}\right) \\
& -\sum_{i=n_{1}+1}^{m}\left(R_{i}+1\right)\left(1+\left(\psi_{i}(\beta)\right)^{v}\right)^{\gamma} \\
& \cdot \log \left(1+\left(\psi_{i}(\beta)\right)^{v}\right) \text {, } \\
& \frac{\partial \ell(\gamma, v, \beta)}{\partial v}=\frac{m}{v}+\sum_{i=1}^{n_{1}} \log y_{i} \\
& +\sum_{i=n_{1}+1}^{m} \log \psi_{i}(\beta)+(\gamma-1) \sum_{i=1}^{n_{1}} \frac{y_{i}^{\nu} \log y_{i}}{1+y_{i}^{\nu}} \\
& +(\gamma-1) \sum_{i=n_{1}+1}^{m} \frac{\left(\psi_{i}(\beta)\right)^{v} \log \psi_{i}(\beta)}{1+\left(\psi_{i}(\beta)\right)^{v}} \\
& -\gamma \sum_{i=1}^{n_{1}}\left(R_{i}+1\right)\left(1+y_{i}^{\nu}\right)^{\gamma-1} y_{i}^{\nu} \log y_{i} \\
& -\gamma \sum_{i=n_{1}+1}^{m}\left(R_{i}+1\right)\left(1+\left(\psi_{i}(\beta)\right)^{\nu}\right)^{\gamma-1}\left(\psi_{i}(\beta)\right)^{\nu} \\
& \text { - } \log \psi_{i}(\beta) \text {, } \\
& \frac{\partial \ell(\gamma, \nu, \beta)}{\partial \beta}=\frac{\left(m-n_{1}\right)}{\beta}+(\nu-1) \sum_{i=n_{1}+1}^{m} \frac{\left(y_{i}-\tau\right)}{\psi_{i}(\beta)} \\
& +\nu(\gamma-1) \sum_{i=n_{1}+1}^{m} \frac{\left(\psi_{i}(\beta)\right)^{\nu-1}\left(y_{i}-\tau\right)}{1+\left(\psi_{i}(\beta)\right)^{\nu}} \\
& -\gamma \nu \sum_{i=n_{1}+1}^{m}\left(R_{i}+1\right)\left[1+\left(\psi_{i}(\beta)\right)^{\nu}\right]^{\gamma-1} \\
& \cdot\left(\psi_{i}(\beta)\right)^{\nu-1}\left(y_{i}-\tau\right) .
\end{aligned}
$$

Now, we have a system of three nonlinear equations in three unknowns $\gamma, \nu$, and $\beta$. It is clear that a closed form solution is very difficult to obtain. Therefore, an iterative procedure such as Newton Raphson can be used to find a numerical solution of the above nonlinear system.

\section{Bayes Estimation}

In this section, the square error loss (SEL) function is considered to obtain BEs of the model parameters $\gamma, v$, and 
$\beta$. Unfortunately, in many cases the BEs cannot be expressed in explicit forms. So, approximate BEs are obtained under noninformative prior (NIP) and informative prior (IP) using Lindley's approximation and Markov chain Monte Carlo (MCMC) method.

4.1. Noninformative Prior. Assume that the parameters $\gamma, \nu$, and $\beta$ are independent and the NIP for each parameter is as follows:

$$
\begin{array}{ll}
\pi(\gamma) \propto \gamma^{-1}, & \gamma>0, \\
\pi(\nu) \propto \nu^{-1}, & \nu>0, \\
\pi(\beta) \propto \beta^{-1}, & \beta>1,
\end{array}
$$

then, the joint NIP of the parameters is given by

$$
\pi_{1}(\gamma, \nu, \beta) \propto(\gamma \nu \beta)^{-1}, \quad \gamma, \nu>0, \beta>1
$$

The joint posterior density function of the parameters $\gamma, \nu$, and $\beta$ can be written from (9) and (15) as

$$
\begin{aligned}
& \pi_{1}^{*}(\gamma, \nu, \beta) \propto L(\gamma, \nu, \beta) \pi_{1}(\gamma, \nu, \beta) \propto(\gamma \nu)^{m-1} \beta^{m-n_{1}-1} \\
& \cdot\left\{\prod_{i=1}^{n_{1}} y_{i}^{\nu-1}\left(1+y_{i}^{\nu}\right)^{(\gamma-1)}\right. \\
&\left.\cdot \exp \left\{\left(R_{i}+1\right)\left(1-\left(1+y_{i}^{\nu}\right)^{\gamma}\right)\right\}\right\} \\
& \cdot\left\{\prod_{i=n_{1}+1}^{m}\left(\psi_{i}(\beta)\right)^{\nu-1}\left(1+\left(\psi_{i}(\beta)\right)^{\nu}\right)^{\gamma-1}\right. \\
&\left.\cdot \exp \left\{\left(R_{i}+1\right)\left(1-\left(1+\left(\psi_{i}(\beta)\right)^{\nu}\right)^{\gamma}\right)\right\}\right\} .
\end{aligned}
$$

Based on SEL function, the Bayes estimator of the function of the parameters $U(\Theta)=U(\gamma, \nu, \beta)$ is

$$
\widetilde{U}(\Theta)=E(U(\Theta))=\int_{\Theta} U(\Theta) \pi_{1}^{*}(\Theta) d \Theta .
$$

Unfortunately, we cannot compute this integral explicitly. Therefore, we adopt two different procedures to approximate this integral; such procedures are Lindley's approximation and MCMC method.

4.1.1. Bayes Estimation Using Lindley's Approximation for NIP. In this sub-subsection, the approximate BEs $\widetilde{\gamma}, \widetilde{\nu}$, and $\widetilde{\beta}$ under SEL function using Lindley's approximation are obtained.

According to Lindley in [10], any ratio of the integral is of the form

$$
\widetilde{U}(\Theta)=E(U(\Theta))=\frac{\int_{\Theta} U(\Theta) e^{\ell(\Theta)+\rho(\Theta)} d \Theta}{\int_{\Theta} e^{\ell(\Theta)+\rho(\Theta)} d \Theta},
$$

where

$U(\Theta)=U\left(\theta_{1}, \theta_{2}, \theta_{3}\right)$ is a function of $\theta_{1}, \theta_{2}$, and $\theta_{3}$ only;

$\ell\left(\theta_{1}, \theta_{2}, \theta_{3}\right)$ is $\log$ of likelihood function;

$\rho\left(\theta_{1}, \theta_{2}, \theta_{3}\right)$ is $\log$ of joint prior of $\theta_{1}, \theta_{2}$, and $\theta_{3}$.

Then, $\widetilde{U}(\Theta)$ can be evaluated as

$$
\begin{aligned}
\widetilde{U}(\Theta) \simeq & U\left(\hat{\theta}_{1}, \hat{\theta}_{2}, \widehat{\theta}_{3}\right) \\
& +\left(U_{1} a_{1}+U_{2} a_{2}+U_{3} a_{3}+a_{4}+a_{5}\right) \\
+ & 0.5\left[\phi_{1}\left(U_{1} \sigma_{11}+U_{2} \sigma_{12}+U_{3} \sigma_{13}\right)\right. \\
& +\phi_{2}\left(U_{1} \sigma_{21}+U_{2} \sigma_{22}+U_{3} \sigma_{23}\right) \\
& \left.+\phi_{3}\left(U_{1} \sigma_{31}+U_{2} \sigma_{32}+U_{3} \sigma_{33}\right)\right]
\end{aligned}
$$

where

$\widehat{\theta}_{i}, i=1,2,3$ are the MLEs of the parameters $\theta_{i}$,

$a_{j}=\sum_{i=1}^{3} \rho_{i} \sigma_{j i}, \quad j=1,2,3$,

$a_{4}=U_{12} \sigma_{12}+U_{13} \sigma_{13}+U_{23} \sigma_{23}$,

$a_{5}=0.5\left(U_{11} \sigma_{11}+U_{22} \sigma_{22}+U_{33} \sigma_{33}\right)$,

$\phi_{1}=\sigma_{11} \ell_{111}+2\left(\sigma_{12} \ell_{121}+\sigma_{13} \ell_{131}+\sigma_{23} \ell_{231}\right)+\sigma_{22} \ell_{221}+$ $\sigma_{33} \ell_{331}$,

$\phi_{2}=\sigma_{11} \ell_{112}+2\left(\sigma_{12} \ell_{122}+\sigma_{13} \ell_{132}+\sigma_{23} \ell_{232}\right)+\sigma_{22} \ell_{222}+$ $\sigma_{33} \ell_{332}$,

$\phi_{3}=\sigma_{11} \ell_{113}+2\left(\sigma_{12} \ell_{123}+\sigma_{13} \ell_{133}+\sigma_{23} \ell_{233}\right)+\sigma_{22} \ell_{223}+$ $\sigma_{33} \ell_{333}$,

$\ell_{i j}=\partial^{2} \ell(\Theta) / \partial \theta_{i} \partial \theta_{j}, \ell_{i j k}=\partial^{3} \ell(\Theta) / \partial \theta_{i} \partial \theta_{j} \partial \theta_{k}$, $U_{i}=\partial U(\Theta) / \partial \theta_{i}, U_{i j}=\partial^{2} U(\Theta) / \partial \theta_{i} \partial \theta_{j}, \rho_{i}=$ $\partial \rho(\Theta) / \partial \theta_{i}, i, j, k=1,2,3$.

$\left(\sigma_{i j}\right)_{3 \times 3}=\left(-\left(\partial^{2} \ell(\Theta) / \partial \theta_{i} \partial \theta_{j}\right)\right)_{3 \times 3}^{-1}$ is the variancecovariance matrix of unknown vector $\Theta=\left(\theta_{1}, \theta_{2}, \theta_{3}\right)$.

Form the prior distribution in (15) and (19), the values of the BEs of various parameters are

$$
\begin{aligned}
\tilde{\gamma}_{L(\mathrm{NIP})} \simeq \hat{\gamma} & -\left(\frac{\sigma_{11}}{\widehat{\gamma}}+\frac{\sigma_{12}}{\widehat{\gamma}}+\frac{\sigma_{13}}{\widehat{\beta}}\right) \\
& +0.5\left(\phi_{1} \sigma_{11}+\phi_{2} \sigma_{12}+\phi_{3} \sigma_{13}\right), \\
\widetilde{\nu}_{L(\mathrm{NIP})} \simeq & \hat{\nu}-\left(\frac{\sigma_{21}}{\widehat{\gamma}}+\frac{\sigma_{22}}{\widehat{\gamma}}+\frac{\sigma_{23}}{\widehat{\beta}}\right) \\
& +0.5\left(\phi_{1} \sigma_{21}+\phi_{2} \sigma_{22}+\phi_{3} \sigma_{23}\right), \\
\widetilde{\beta}_{L(\mathrm{NIP})} \simeq & \widehat{\beta}-\left(\frac{\sigma_{31}}{\widehat{\gamma}}+\frac{\sigma_{32}}{\widehat{\nu}}+\frac{\sigma_{33}}{\widehat{\beta}}\right) \\
& +0.5\left(\phi_{1} \sigma_{31}+\phi_{2} \sigma_{32}+\phi_{3} \sigma_{33}\right) .
\end{aligned}
$$


TABle 1: The progressive censoring schemes used in the Monte Carlo simulation study.

\begin{tabular}{|c|c|c|c|c|c|c|c|}
\hline$n$ & $m$ & C.S & $\left(R_{1}, \ldots, R_{m}\right)$ & C.S & $\left(R_{1}, \ldots, R_{m}\right)$ & C.S & $\left(R_{1}, \ldots, R_{m}\right)$ \\
\hline 15 & 9 & {$[1]$} & $R_{i}= \begin{cases}1 & i=3, \ldots, 8 \\
0 & i=1,2,9\end{cases}$ & {$[2]$} & $R_{1}=6, R_{i}=0, i \neq 1$ & {$[3]$} & $R_{9}=6, R_{i}=0, i \neq 9$ \\
\hline 15 & 12 & {$[4]$} & $\begin{cases}1 & i=4, \ldots, 6 \\
0 & \text { otherwise }\end{cases}$ & {$[5]$} & $R_{1}=3, R_{i}=0, i \neq 1$ & {$[6]$} & $R_{12}=3, R_{i}=0, i \neq 12$ \\
\hline 25 & 15 & [7] & $\begin{cases}1 & i=2, \ldots, 11 \\
0 & \text { otherwise }\end{cases}$ & {$[8]$} & $R_{1}=10, R_{i}=0, i \neq 1$ & [9] & $R_{15}=10, R_{i}=0, i \neq 15$ \\
\hline 25 & 20 & [10] & $\begin{cases}1 & i=8, \ldots, 12 \\
0 & \text { otherwise }\end{cases}$ & [11] & $R_{1}=5, R_{i}=0, i \neq 1$ & {$[12]$} & $R_{20}=5, R_{i}=0, i \neq 20$ \\
\hline 50 & 30 & [13] & $\begin{cases}1 & i=6, \ldots, 25 \\
0 & \text { otherwise }\end{cases}$ & {$[14]$} & $R_{1}=20, R_{i}=0, i \neq 1$ & {$[15]$} & $R_{30}=20, R_{i}=0, i \neq 30$ \\
\hline 50 & 40 & [16] & $\begin{array}{ll}1 & i=16, \ldots, 25 \\
0 & \text { otherwise }\end{array}$ & [17] & $R_{1}=10, R_{i}=0, i \neq 1$ & {$[18]$} & $R_{40}=10, R_{i}=0, i \neq 40$ \\
\hline
\end{tabular}

TABLE 2: SSPALT simulation data with true values $\gamma=0.5, \quad v=0.7$, $\beta=1.1$, and $\tau=0.9$.

\begin{tabular}{lc}
\hline $\begin{array}{l}\text { Failure times under normal } \\
\text { condition }\end{array}$ & $\begin{array}{c}\text { Failure times under accelerated } \\
\text { condition }\end{array}$ \\
\hline $0.0349,0.0362,0.0895,0.1177$, & $1.4067,1.8032,1.8854,2.1311$, \\
$0.1686,0.1770,0.4748$ & $9.9164,11.0643,12.5569,13.2147$, \\
& 49.5808 \\
\hline
\end{tabular}

4.1.2. Bayesian Estimation Using MCMC Method for NIP. In this sub-subsection, MCMC method is considered to generate samples from the posterior distribution and then compute the BEs of $\gamma, \nu$, and $\beta$ under SSPALT using progressive type-II censoring.

From the joint posterior density function in (16), the conditional posterior distributions of $\gamma, \nu$, and $\beta$ can be written, respectively, as

$$
\begin{aligned}
& \pi_{1}^{*}(\gamma \mid \nu, \beta) \propto \gamma^{m-1} \\
& \cdot\left\{\prod_{i=1}^{n_{1}}\left(1+y_{i}^{\nu}\right)^{\gamma} \exp \left\{-\left(R_{i}+1\right)\left(1+y_{i}^{\nu}\right)^{\gamma}\right\}\right\} \\
& \cdot\left\{\prod_{i=n_{1}+1}^{m}\left(1+\left(\psi_{i}(\beta)\right)^{\nu}\right)^{\gamma}\right. \\
&\left.\cdot \exp \left\{-\left(R_{i}+1\right)\left(1+\left(\psi_{i}(\beta)\right)^{\nu}\right)^{\gamma}\right\}\right\},
\end{aligned}
$$

$$
\begin{aligned}
\pi_{1}^{*}(\nu \mid \gamma, \beta) \propto & \nu^{m-1} \\
& \cdot\left\{\prod_{i=1}^{n_{1}} y_{i}^{\nu}\left(1+y_{i}^{\nu}\right)^{\gamma-1}\right. \\
& \left.\cdot \exp \left\{-\left(R_{i}+1\right)\left(1+y_{i}^{\nu}\right)^{\gamma}\right\}\right\} \\
& \cdot\left\{\prod_{i=n_{1}+1}^{m}\left(\psi_{i}(\beta)\right)^{\nu}\left(1+\left(\psi_{i}(\beta)\right)^{\nu}\right)^{\gamma-1}\right. \\
& \left.\cdot \exp \left\{-\left(R_{i}+1\right)\left(1+\left(\psi_{i}(\beta)\right)^{\nu}\right)^{\gamma}\right\}\right\},
\end{aligned}
$$

$$
\begin{aligned}
\pi_{1}^{*}(\beta \mid \gamma, \nu) \propto & \beta^{m-n_{1}-1} \\
& \cdot \prod_{i=n_{1}+1}^{m}\left(\psi_{i}(\beta)\right)^{\nu-1}\left(1+\left(\psi_{i}(\beta)\right)^{\nu}\right)^{\gamma-1} \\
& \quad \cdot \exp \left\{-\left(R_{i}+1\right)\left(1+\left(\psi_{i}(\beta)\right)^{\nu}\right)^{\gamma}\right\} .
\end{aligned}
$$

The conditional posterior distributions of $\gamma, \nu$, and $\beta$ in (23), (24) and (25) cannot be reduced analytically to well known distribution, but the plot of them shows that they are similar to normal distribution. So, to generate random samples from this distribution, we use the Metropolis method with normal proposal distribution; see Metropolis et al. [11].

The following algorithm is proposed to generate $\gamma, \nu$, and $\beta$ from the posterior distribution and then obtain the BEs. 
TABLE 3: MSEs of the estimates of $\gamma, \nu$, and $\beta$ with population parameter values: $(\gamma=0.5, \nu=0.7$, and $\beta=1.1), \tau=0.9, n=30, m=20$, and $R_{1}=10, R_{i}=0, i=2,3, \ldots, 20$.

\begin{tabular}{lcccc}
\hline $\operatorname{MSE}\left(\widehat{\gamma}_{\text {MLE }}\right)$ & $\operatorname{MSE}\left(\widetilde{\gamma}_{L(\mathrm{NIP})}\right)$ & $\operatorname{MSE}\left(\widetilde{\gamma}_{L(\mathrm{IP})}\right)$ & $\operatorname{MSE}\left(\widetilde{\gamma}_{\mathrm{MC}(\mathrm{NIP})}\right)$ & $\operatorname{MSE}\left(\widetilde{\gamma}_{\mathrm{MC}(\mathrm{IP})}\right)$ \\
$\operatorname{MSE}\left(\widehat{\nu}_{\mathrm{MLE}}\right)$ & $\operatorname{MSE}\left(\widetilde{\nu}_{L(\mathrm{NIP})}\right)$ & $\operatorname{MSE}\left(\widetilde{\nu}_{L(\mathrm{IP})}\right)$ & $\operatorname{MSE}\left(\widetilde{\nu}_{\mathrm{MC}(\mathrm{NIP})}\right)$ & $\operatorname{MSE}\left(\widetilde{\nu}_{\mathrm{MC}(\mathrm{IP})}\right)$ \\
$\operatorname{MSE}\left(\widehat{\beta}_{\mathrm{MLE}}\right)$ & $\operatorname{MSE}\left(\widetilde{\beta}_{L(\mathrm{NIP})}\right)$ & $\operatorname{MSE}\left(\widetilde{\beta}_{L(\mathrm{IP})}\right)$ & 0.0031 & 0.0025 \\
\hline 0.0056 & 0.0043 & 0.0032 & 0.0041 & 0.0031 \\
0.0009 & 0.0014 & 0.0036 & 0.07854 & 0.07325 \\
0.0435 & 0.0223 & 0.08164 & $\left.\widetilde{\beta}_{\mathrm{MC}(\mathrm{NIP})}\right)$ & \\
\hline
\end{tabular}

\section{Algorithm 1.}

Step 1. Start with $\gamma^{(0)}=\widehat{\gamma}_{\mathrm{MLE}}, v^{(0)}=\widehat{\nu}_{\mathrm{MLE}}$, and $\beta^{(0)}=\widehat{\beta}_{\mathrm{MLE}}$.

Step 2. Set $i=1$.

Step 3. Generate $\gamma^{*}$ from proposal distribution $\mathbf{N}\left(\gamma^{(i-1)}\right.$, $\left.\operatorname{var}\left(\gamma^{(i-1)}\right)\right)$.

Step 4. Calculate the acceptance probability

$$
r\left(\gamma^{(i-1)} \mid \gamma^{*}\right)=\min \left[1, \frac{\pi_{1}^{*}\left(\gamma^{*} \mid \nu^{(i-1)}, \beta^{(i-1)}\right)}{\pi_{1}^{*}\left(\gamma^{(i-1)} \mid \nu^{(i-1)}, \beta^{(i-1)}\right)}\right] .
$$

Step 5. Generate $U \sim U(0,1)$.

Step 6. If $U \leq r\left(\gamma^{(i-1)} \mid \gamma^{*}\right)$, accept the proposal distribution and set $\gamma^{(i)}=\gamma^{*}$. Otherwise, reject the proposal distribution and set $\gamma^{(i)}=\gamma^{(i-1)}$.

Step 7. To generate $\nu^{*}$ do the Steps 2-6 for $\nu$ not $\gamma$.

Step 8. To generate $\beta^{*}$ do the Steps $2-6$ for $\beta$ not $\gamma$.

Step 9. Set $i=i+1$.

Step 10. Repeat Steps 3-9 N times.

Step 11. Obtain the BEs of $\gamma, \nu$, and $\beta$ using MCMC under SEL function as

$$
\begin{aligned}
& \widetilde{\gamma}_{\mathrm{MC}(\mathrm{NIP})}=\frac{1}{N-M} \sum_{i=M+1}^{N} \gamma^{(i)} \\
& \widetilde{\nu}_{\mathrm{MC}(\mathrm{NIP})}=\frac{1}{N-M} \sum_{i=M+1}^{N} \gamma^{(i)} \\
& \widetilde{\beta}_{\mathrm{MC}(\mathrm{NIP})}=\frac{1}{N-M} \sum_{i=M+1}^{N} \beta^{(i)}
\end{aligned}
$$

4.2. Informative Prior. Assume that the parameters $\gamma$ and $\nu$ are dependent have prior density $\gamma \mid \nu \sim \operatorname{Gamma}(\mu, \nu)$, $\nu \sim \operatorname{Exp}(\lambda)$ and $\beta$ is independent of them with NIP as $\pi(\beta) \propto$ $\beta^{-1}, \beta>1$; then, the joint IP of the parameters is given by

$$
\pi_{2}(\gamma, \nu, \beta) \propto \beta^{-1} \nu^{-\mu} \gamma^{\mu-1} e^{-(\nu / \lambda+\gamma / \nu)}, \quad \gamma, \nu>0, \beta>1 .
$$

TABLE 4: 95\% approximate and bootstrap CIs and their lengths of the parameters $\gamma, \nu$, and $\beta$ with population parameter values $\gamma=0.5$, $\nu=0.7, \beta=1.1$, and $\tau=0.9$.

\begin{tabular}{lccc}
\hline \multicolumn{2}{c}{ CIs } & \multicolumn{2}{c}{ Lengths of CIs } \\
\hline Approx. $\mathrm{CI}(\gamma)$ & Boot $\mathrm{CI}(\gamma)$ & Approx. $\mathrm{CI}(\gamma)$ & Boot $\mathrm{CI}(\gamma)$ \\
Approx. $\mathrm{CI}(\nu)$ & Boot $\mathrm{CI}(\nu)$ & Approx. CI $(\nu)$ & Boot $\mathrm{CI}(\nu)$ \\
Approx. $\mathrm{CI}(\beta)$ & Boot $\mathrm{CI}(\beta)$ & Approx. $\mathrm{CI}(\beta)$ & Boot $\mathrm{CI}(\beta)$ \\
\hline$(0.2959,0.8539)$ & $(0.3343,0.7175)$ & 0.5580 & 0.3831 \\
$(0.4159,1.0467)$ & $(0.5154,0.9153)$ & 0.6307 & 0.3998 \\
$(0.4400,2.2226)$ & $(0.3922,2.1087)$ & 2.2226 & 1.7164 \\
\hline
\end{tabular}

The joint posterior density function of the parameters $\gamma, \nu$, and $\beta$ can be written from (9) and (28) as

$$
\begin{aligned}
& \pi_{2}^{*}(\gamma, \nu, \beta) \propto \gamma^{m+\mu-1} \nu^{m-\mu} \beta^{m-n_{1}-1} e^{-(\nu / \lambda+\gamma / \nu)} \\
& \cdot\left\{\prod_{i=1}^{n_{1}} y_{i}^{\nu-1}\left(1+y_{i}^{\nu}\right)^{\gamma-1}\right. \\
&\left.\cdot \exp \left\{\left(R_{i}+1\right)\left(1-\left(1+y_{i}^{\nu}\right)^{\gamma}\right)\right\}\right\} \\
& \cdot\left\{\prod_{i=n_{1}+1}^{m}\left(\psi_{i}(\beta)\right)^{\nu-1}\left(1+\left(\psi_{i}(\beta)\right)^{\nu}\right)^{\gamma-1}\right. \\
&\left.\cdot \exp \left\{\left(R_{i}+1\right)\left(1-\left(1+\left(\psi_{i}(\beta)\right)^{\nu}\right)^{\gamma}\right)\right\}\right\} .
\end{aligned}
$$

Based on SEL function, the Bayes estimator of the function of the parameters $U(\Theta)=U(\gamma, \nu, \beta)$ is

$$
\widetilde{U}(\Theta)=\int_{\Theta} U(\Theta) \pi_{2}^{*}(\Theta) d \Theta .
$$

Unfortunately, this integral cannot be reduced to a closed form. Therefore, we adopt two different procedures to approximate this integral; such procedures are Lindley's approximation and MCMC method.

4.2.1. Lindley's Approximation of Bayes Estimation for IP. In this sub-subsection, Lindley's approximation is used to obtain BEs of $\gamma, \nu$, and $\beta$ under SEL function. 
TABLE 5: MSEs of the estimates of $\gamma, \nu$, and $\beta$ with true values $\gamma=0.5, \nu=0.7, \beta=1.1$, and $\tau=0.9$.

\begin{tabular}{|c|c|c|c|c|c|c|c|c|}
\hline$n$ & $m$ & $n_{1}$ & C.S & $\begin{array}{l}\operatorname{MSE}\left(\widehat{\gamma}_{\mathrm{MLE}}\right) \\
\operatorname{MSE}\left(\widehat{\gamma}_{\mathrm{MLE}}\right) \\
\operatorname{MSE}\left(\widehat{\beta}_{\mathrm{MLE}}\right)\end{array}$ & $\begin{array}{l}\operatorname{MSE}\left(\widetilde{\gamma}_{L(\mathrm{NIP})}\right) \\
\operatorname{MSE}\left(\widetilde{\nu}_{L(\mathrm{NIP})}\right) \\
\operatorname{MSE}\left(\widetilde{\beta}_{L(\mathrm{NIP})}\right)\end{array}$ & $\begin{array}{l}\operatorname{MSE}\left(\widetilde{\gamma}_{L(\mathrm{IP})}\right) \\
\operatorname{MSE}\left(\widetilde{\gamma}_{L(\mathrm{IP})}\right) \\
\operatorname{MSE}\left(\widetilde{\beta}_{L(\mathrm{IP})}\right)\end{array}$ & $\begin{array}{l}\operatorname{MSE}\left(\widetilde{\gamma}_{\mathrm{MC}(\mathrm{NIP})}\right) \\
\operatorname{MSE}\left(\widetilde{\nu}_{\mathrm{MC}(\mathrm{NIP})}\right) \\
\operatorname{MSE}\left(\widetilde{\beta}_{\mathrm{MC}(\mathrm{NIP})}\right)\end{array}$ & $\begin{array}{l}\operatorname{MSE}\left(\widetilde{\gamma}_{\mathrm{MC}(\mathrm{IP})}\right) \\
\operatorname{MSE}\left(\widetilde{\nu}_{\mathrm{MC}(\mathrm{IP})}\right) \\
\operatorname{MSE}\left(\widetilde{\beta}_{\mathrm{MC}(\mathrm{IP})}\right)\end{array}$ \\
\hline \multirow{9}{*}{15} & \multirow{9}{*}{9} & \multirow{9}{*}{3} & \multirow{3}{*}[1]{} & 0.0723 & 0.0281 & 0.0239 & 0.0176 & 0.0178 \\
\hline & & & & 0.5822 & 0.0702 & 0.0761 & 0.0471 & 0.0447 \\
\hline & & & & 0.7012 & 0.3910 & 0.4080 & 0.6077 & 0.6077 \\
\hline & & & \multirow{3}{*}[2]{} & 0.0424 & 0.0212 & 0.0171 & 0.0173 & 0.0162 \\
\hline & & & & 0.3577 & 0.0647 & 0.0718 & 0.0384 & 0.0396 \\
\hline & & & & 0.7016 & 0.3738 & 0.3838 & 0.5969 & 0.5835 \\
\hline & & & \multirow{3}{*}{ [3] } & 0.0442 & 0.0223 & 0.0215 & 0.0191 & 0.0175 \\
\hline & & & & 0.3798 & 0.0690 & 0.0725 & 0.0495 & 0.0537 \\
\hline & & & & 0.7121 & 0.4462 & 0.5315 & 0.6221 & 0.5825 \\
\hline \multirow{9}{*}{15} & \multirow{9}{*}{12} & \multirow{9}{*}{4} & \multirow{3}{*}[4]{} & 0.0378 & 0.0217 & 0.0187 & 0.0125 & 0.0125 \\
\hline & & & & 0.5772 & 0.0654 & 0.0688 & 0.0306 & 0.0311 \\
\hline & & & & 0.6205 & 0.3270 & 0.3917 & 0.4611 & 0.4374 \\
\hline & & & \multirow{3}{*}{ [5] } & 0.0243 & 0.0211 & 0.0157 & 0.0122 & 0.0119 \\
\hline & & & & 0.2604 & 0.0639 & 0.0674 & 0.0284 & 0.0280 \\
\hline & & & & 0.5942 & 0.2668 & 0.3698 & 0.4408 & 0.5013 \\
\hline & & & \multirow{3}{*}[6]{} & 0.0300 & 0.0219 & 0.0193 & 0.0138 & 0.0131 \\
\hline & & & & 0.3727 & 0.0654 & 0.0718 & 0.0358 & 0.0327 \\
\hline & & & & 0.6166 & 0.3043 & 0.4191 & 0.4203 & 0.4414 \\
\hline \multirow{9}{*}{25} & \multirow{9}{*}{15} & \multirow{9}{*}{5} & \multirow{3}{*}{ [7] } & 0.0288 & 0.0212 & 0.0207 & 0.0111 & 0.0110 \\
\hline & & & & 0.0870 & 0.0589 & 0.0501 & 0.0225 & 0.0244 \\
\hline & & & & 0.5576 & 0.4094 & 0.3854 & 0.3966 & 0.4056 \\
\hline & & & \multirow{3}{*}[8]{} & 0.0178 & 0.0188 & 0.0145 & 0.0107 & 0.0097 \\
\hline & & & & 0.0710 & 0.0568 & 0.0476 & 0.0200 & 0.0203 \\
\hline & & & & 0.6650 & 0.2650 & 0.2656 & 0.3965 & 0.4154 \\
\hline & & & \multirow{3}{*}{ [9] } & 0.0354 & 0.0192 & 0.0212 & 0.0128 & 0.0120 \\
\hline & & & & 0.0903 & 0.0558 & 0.0498 & 0.0299 & 0.0277 \\
\hline & & & & 0.6547 & 0.3718 & 0.4065 & 0.3766 & 0.4158 \\
\hline \multirow{9}{*}{25} & \multirow{9}{*}{20} & & \multirow{3}{*}[10]{} & 0.0200 & 0.0189 & 0.0155 & 0.0081 & 0.0075 \\
\hline & & & & 0.0685 & 0.0491 & 0.0462 & 0.0180 & 0.01776 \\
\hline & & & & 0.5491 & 0.3811 & 0.3620 & 0.3128 & 0.2705 \\
\hline & & & & 0.0197 & 0.0159 & 0.0138 & 0.0073 & 0.0071 \\
\hline & & 7 & [11] & 0.0682 & 0.0453 & 0.0421 & 0.0174 & 0.0165 \\
\hline & & & & 0.6551 & 0.2645 & 0.2535 & 0.3107 & 0.2921 \\
\hline & & & & 0.0236 & 0.0191 & 0.0151 & 0.0093 & 0.0083 \\
\hline & & & [12] & 0.0787 & 0.0465 & 0.0489 & 0.0189 & 0.0199 \\
\hline & & & & 0.5472 & 0.3656 & 0.3391 & 0.2980 & 0.2881 \\
\hline
\end{tabular}


TABle 5: Continued.

\begin{tabular}{|c|c|c|c|c|c|c|c|c|}
\hline$n$ & $m$ & $n_{1}$ & C.S & $\begin{array}{l}\operatorname{MSE}\left(\widehat{\gamma}_{\mathrm{MLE}}\right) \\
\operatorname{MSE}\left(\widehat{\nu}_{\mathrm{MLE}}\right) \\
\operatorname{MSE}\left(\widehat{\beta}_{\mathrm{MLE}}\right)\end{array}$ & $\begin{array}{l}\operatorname{MSE}\left(\tilde{\gamma}_{L(\mathrm{NIP})}\right) \\
\operatorname{MSE}\left(\widetilde{\nu}_{L(\mathrm{NIP})}\right) \\
\operatorname{MSE}\left(\tilde{\beta}_{L(\mathrm{NIP})}\right)\end{array}$ & $\begin{array}{l}\operatorname{MSE}\left(\widetilde{\gamma}_{L(\mathrm{IP})}\right) \\
\operatorname{MSE}\left(\widetilde{v}_{L(\mathrm{IP})}\right) \\
\operatorname{MSE}\left(\widetilde{\beta}_{L(\mathrm{IP})}\right) \\
\end{array}$ & $\begin{array}{l}\operatorname{MSE}\left(\widetilde{\gamma}_{\mathrm{MC}(\mathrm{NIP})}\right) \\
\operatorname{MSE}\left(\widetilde{\nu}_{\mathrm{MC}(\mathrm{NIP})}\right) \\
\operatorname{MSE}\left(\widetilde{\beta}_{\mathrm{MC}(\mathrm{NIP})}\right)\end{array}$ & $\begin{array}{l}\operatorname{MSE}\left(\widetilde{\gamma}_{\mathrm{MC}(\mathrm{IP})}\right) \\
\operatorname{MSE}\left(\widetilde{\nu}_{\mathrm{MC}(\mathrm{IP})}\right) \\
\operatorname{MSE}\left(\widetilde{\beta}_{\mathrm{MC}(\mathrm{IP})}\right)\end{array}$ \\
\hline \multirow{9}{*}{50} & \multirow{9}{*}{30} & \multirow{9}{*}{10} & \multirow{3}{*}{ [13] } & 0.0223 & 0.0180 & 0.0133 & 0.0061 & 0.0066 \\
\hline & & & & 0.0255 & 0.0366 & 0.0372 & 0.0124 & 0.0120 \\
\hline & & & & 0.4730 & 0.4049 & 0.3208 & 0.2269 & 0.2256 \\
\hline & & & \multirow{3}{*}[14]{} & 0.0142 & 0.0149 & 0.0107 & 0.0056 & 0.0056 \\
\hline & & & & 0.0295 & 0.0308 & 0.0284 & 0.0103 & 0.0100 \\
\hline & & & & 0.5914 & 0.2250 & 0.2531 & 0.2258 & 0.2164 \\
\hline & & & \multirow{3}{*}[15]{} & 0.0145 & 0.0184 & 0.0150 & 0.0078 & 0.0076 \\
\hline & & & & 0.0266 & 0.0382 & 0.0323 & 0.0145 & 0.0144 \\
\hline & & & & 0.6192 & 0.3616 & 0.3361 & 0.2259 & 0.2188 \\
\hline \multirow{9}{*}{50} & \multirow{9}{*}{40} & \multirow{9}{*}{15} & \multirow{3}{*}[16]{} & 0.0175 & 0.0160 & 0.0107 & 0.0049 & 0.0045 \\
\hline & & & & 0.0228 & 0.0332 & 0.0322 & 0.0085 & 0.0094 \\
\hline & & & & 0.5199 & 0.2791 & 0.2576 & 0.1657 & 0.1676 \\
\hline & & & \multirow{3}{*}{ [17] } & 0.0153 & 0.0124 & 0.0087 & 0.0042 & 0.0043 \\
\hline & & & & 0.0245 & 0.0275 & 0.0273 & 0.0076 & 0.0079 \\
\hline & & & & 0.5537 & 0.2213 & 0.2335 & 0.1790 & 0.1804 \\
\hline & & & \multirow{3}{*}[18]{} & 0.0128 & 0.0163 & 0.0098 & 0.0050 & 0.0048 \\
\hline & & & & 0.0353 & 0.0332 & 0.0274 & 0.0092 & 0.0102 \\
\hline & & & & 0.5110 & 0.3434 & 0.3016 & 0.1615 & 0.1510 \\
\hline
\end{tabular}

From (19) and (28), the BEs of various parameters are

$$
\begin{aligned}
\tilde{\gamma}_{L(\mathrm{IP})} \simeq & \widehat{\gamma}+\left(\frac{\mu-1}{\widehat{\gamma}}-\frac{1}{\widehat{\nu}}\right) \sigma_{11}+\left(\frac{\widehat{\gamma}}{\widehat{\nu}^{2}}-\frac{\mu}{\widehat{\nu}}-\frac{1}{\lambda}\right) \sigma_{12} \\
& -\frac{\sigma_{13}}{\widehat{\beta}}+0.5\left(\phi_{1} \sigma_{11}+\phi_{2} \sigma_{12}+\phi_{3} \sigma_{13}\right), \\
\widetilde{\nu}_{L(\mathrm{IP})} \simeq & \widehat{\nu}+\left(\frac{\mu-1}{\widehat{\gamma}}-\frac{1}{\widehat{\nu}}\right) \sigma_{21}+\left(\frac{\widehat{\gamma}}{\widehat{\nu}^{2}}-\frac{\mu}{\widehat{\nu}}-\frac{1}{\lambda}\right) \sigma_{22} \\
& -\frac{\sigma_{23}}{\widehat{\beta}}+0.5\left(\phi_{1} \sigma_{12}+\phi_{2} \sigma_{22}+\phi_{3} \sigma_{23}\right), \\
\simeq & \widehat{\beta}+\left(\frac{\mu-1}{\widehat{\gamma}}-\frac{1}{\widehat{\gamma}}\right) \sigma_{31}+\left(\frac{\widehat{\gamma}}{\widehat{\gamma}^{2}}-\frac{\mu}{\widehat{\nu}}-\frac{1}{\lambda}\right) \sigma_{32} \\
& -\frac{\sigma_{33}}{\widehat{\beta}}+0.5\left(\phi_{1} \sigma_{31}+\phi_{2} \sigma_{32}+\phi_{3} \sigma_{33}\right) .
\end{aligned}
$$

4.2.2. Bayesian Estimation Using MCMC Method for IP. In this sub-subsection, the BEs of $\gamma, \nu$, and $\beta$ under SSPALT using progressive type-II censoring for IP case are obtained.
From the joint posterior density function in (29), the conditional posterior distributions of $\gamma, \nu$, and $\beta$ can be written, respectively, as

$$
\begin{aligned}
& \pi_{2}^{*}(\gamma \mid \nu, \beta) \propto \gamma^{m+\mu-1} e^{-\gamma / \nu} \\
& \cdot\left\{\prod_{i=1}^{n_{1}}\left(1+y_{i}^{\nu}\right)^{\gamma} \exp \left\{-\left(R_{i}+1\right)\left(1+y_{i}^{\nu}\right)^{\gamma}\right\}\right\} \\
& \cdot\left\{\prod_{i=n_{1}+1}^{m}\left(1+\left(\psi_{i}(\beta)\right)^{\nu}\right)^{\gamma}\right. \\
&\left.\cdot \exp \left\{-\left(R_{i}+1\right)\left(1+\left(\psi_{i}(\beta)\right)^{\nu}\right)^{\gamma}\right\}\right\},
\end{aligned}
$$

$$
\pi_{2}^{*}(\nu \mid \gamma, \beta) \propto \nu^{m-\mu} e^{-(\nu / \lambda+\gamma / v)}
$$

$$
\left\{\prod_{i=1}^{n_{1}} y_{i}^{\nu}\left(1+y_{i}^{\nu}\right)^{\gamma-1}\right.
$$

$$
\left.\cdot \exp \left\{-\left(R_{i}+1\right)\left(1+y_{i}^{\nu}\right)^{\gamma}\right\}\right\}
$$


TABLE 6: Approximate CIs, bootstrap CIs, and their lengths of the parameters $\gamma, \nu$, and $\beta$ with true values $\gamma=0.5, \nu=0.7, \beta=1.1$, and $\tau=0.9$.

\begin{tabular}{|c|c|c|c|c|c|c|}
\hline \multirow{4}{*}{$n$} & \multirow{4}{*}{$m$} & \multirow{4}{*}{ C.S } & \multicolumn{2}{|c|}{ CIs } & \multicolumn{2}{|c|}{ Lengths of CIs } \\
\hline & & & Approx. $\mathrm{CI}(\gamma)$ & Boot $\mathrm{CI}(\gamma)$ & Approx. CI $(\gamma)$ & $\operatorname{Boot} \mathrm{CI}(\gamma)$ \\
\hline & & & Approx. $\mathrm{CI}(\nu)$ & Boot $\mathrm{CI}(\nu)$ & Approx. $\mathrm{CI}(\nu)$ & Boot $\mathrm{CI}(\nu)$ \\
\hline & & & Approx. $\operatorname{CI}(\beta)$ & Boot $\mathrm{CI}(\beta)$ & Approx. $\operatorname{CI}(\beta)$ & Boot $\mathrm{CI}(\beta)$ \\
\hline \multirow{9}{*}{15} & \multirow{9}{*}{9} & \multirow{3}{*}[1]{} & $(0.1489,0.7336)$ & $(0.2118,0.7733)$ & 0.5847 & 0.5615 \\
\hline & & & $(0.3260,1.4214)$ & $(0.4495,1.4362)$ & 1.0954 & 0.9867 \\
\hline & & & $(0.264,3.4898)$ & $(0.4590,2.5987)$ & 3.2257 & 2.1397 \\
\hline & & \multirow{3}{*}[2]{} & $(0.1980,0.7669)$ & $(0.2279,0.7145)$ & 0.5689 & 0.4865 \\
\hline & & & $(0.3777,1.3116)$ & $(0.4370,1.4153)$ & 0.9338 & 0.9783 \\
\hline & & & $(0.1832,3.1164)$ & $(0.3830,2.5063)$ & 2.9332 & 2.1233 \\
\hline & & \multirow{3}{*}[3]{} & $(0.1535,0.7461)$ & $(0.2099,0.7724)$ & 0.5926 & 0.5625 \\
\hline & & & $(0.3293,1.3863)$ & $(0.4247,1.4169)$ & 1.0569 & 0.9922 \\
\hline & & & $(0.0386,3.3041)$ & $(0.4703,2.5948)$ & 3.2654 & 2.1245 \\
\hline \multirow{9}{*}{15} & \multirow{9}{*}{12} & \multirow{3}{*}[4]{} & $(0.2115,0.7131)$ & $(0.2333,0.6369)$ & 0.5016 & 0.4036 \\
\hline & & & $(0.3766,1.3059)$ & $(0.4388,1.3854)$ & 0.9293 & 0.9466 \\
\hline & & & $(0.3775,3.2869)$ & $(0.3155,2.4013)$ & 2.9094 & 2.0858 \\
\hline & & \multirow{3}{*}[5]{} & $(0.2342,0.7623)$ & $(0.2449,0.7071)$ & 0.5281 & 0.4621 \\
\hline & & & $(0.4032,1.2425)$ & $(0.4430,1.2969)$ & 0.8392 & 0.8539 \\
\hline & & & $(0.1291,2.8591)$ & $(0.2441,2.3952)$ & 2.7300 & 2.1511 \\
\hline & & \multirow{3}{*}[6]{} & $(0.2151,0.7266)$ & $(0.2543,0.6699)$ & 0.5114 & 0.4156 \\
\hline & & & $(0.3851,1.2612)$ & $(0.4455,1.3172)$ & 0.8760 & 0.8717 \\
\hline & & & $(0.1255,3.0455)$ & $(0.3474,2.4387)$ & 2.9200 & 2.0913 \\
\hline \multirow{9}{*}{25} & \multirow{9}{*}{15} & \multirow{3}{*}[7]{} & $(0.2230,0.6928)$ & $(0.2898,0.6862)$ & 0.4698 & 0.3964 \\
\hline & & & $(0.4230,1.1528)$ & $(0.4555,1.1792)$ & 0.7297 & 0.7237 \\
\hline & & & $(0.1757,3.0817)$ & $(0.4616,2.3486)$ & 2.9060 & 1.8870 \\
\hline & & \multirow{3}{*}[8]{} & $(0.2527,0.7250)$ & $(0.2686,0.6988)$ & 0.4723 & 0.4302 \\
\hline & & & $(0.4091,1.1189)$ & $(0.4626,1.1053)$ & 0.7097 & 0.6426 \\
\hline & & & $(0.0479,2.9299)$ & $(0.2599,2.1087)$ & 2.8820 & 1.8487 \\
\hline & & \multirow{3}{*}[9]{} & $(0.1888,0.6847)$ & $(0.2194,0.6154)$ & 0.4958 & 0.3959 \\
\hline & & & $(0.3968,1.1823)$ & $(0.4807,1.1153)$ & 0.7855 & 0.6346 \\
\hline & & & $(0.2021,3.1165)$ & $(0.4966,2.3013)$ & 2.9144 & 1.8047 \\
\hline \multirow{9}{*}{25} & \multirow{9}{*}{20} & \multirow{3}{*}[10]{} & $(0.2600,0.6728)$ & $(0.2147,0.5963)$ & 0.4127 & 0.3816 \\
\hline & & & $(0.4214,1.0669)$ & $(0.4724,1.1021)$ & 0.6453 & 0.6297 \\
\hline & & & $(0.1008,2.9044)$ & $(0.3237,2.1261)$ & 2.8036 & 1.8024 \\
\hline & & \multirow{3}{*}[11]{} & $(0.2608,0.7478)$ & $(0.3169,0.7102)$ & 0.4397 & 0.3933 \\
\hline & & & $(0.3996,1.0844)$ & $(0.4630,0.9758)$ & 0.6848 & 0.5128 \\
\hline & & & $(0.1159,2.9285)$ & $(0.2506,2.0141)$ & 2.8126 & 1.7635 \\
\hline & & \multirow{3}{*}[12]{} & $(0.2594,0.6774)$ & $(0.2583,0.5887)$ & 0.4179 & 0.3304 \\
\hline & & & $(0.4163,1.0889)$ & $(0.4536,1.0658)$ & 0.6725 & 0.6122 \\
\hline & & & $(0.0042,2.8281)$ & $(0.3537,2.1345)$ & 2.8239 & 1.7808 \\
\hline
\end{tabular}


TABle 6: Continued.

\begin{tabular}{|c|c|c|c|c|c|c|}
\hline \multirow{4}{*}{$n$} & \multirow{4}{*}{$m$} & \multirow{4}{*}{ C.S } & \multicolumn{2}{|c|}{ CIs } & \multicolumn{2}{|c|}{ Lengths of CIs } \\
\hline & & & Approx. $\mathrm{CI}(\gamma)$ & Boot $\mathrm{CI}(\gamma)$ & Approx. $\mathrm{CI}(\gamma)$ & $\operatorname{Boot} \mathrm{CI}(\gamma)$ \\
\hline & & & Approx. CI $(\nu)$ & Boot $\mathrm{CI}(\nu)$ & Approx. CI $(\nu)$ & $\operatorname{Boot} \mathrm{CI}(\nu)$ \\
\hline & & & Approx. $\mathrm{CI}(\beta)$ & Boot $\mathrm{CI}(\beta)$ & Approx. $\mathrm{CI}(\beta)$ & Boot $\mathrm{CI}(\beta)$ \\
\hline \multirow{9}{*}{50} & \multirow{9}{*}{30} & \multirow{3}{*}[13]{} & $(0.2610,0.5761)$ & $(0.2786,0.5769)$ & 0.3150 & 0.2983 \\
\hline & & & $(0.4734,1.0151)$ & $(0.5172,0.9153)$ & 0.5416 & 0.3975 \\
\hline & & & $(0.2034,2.8585)$ & $(0.5499,2.1088)$ & 2.6551 & 1.5589 \\
\hline & & \multirow{3}{*}[14]{} & $(0.3237,0.6703)$ & $(0.2911,0.6692)$ & 0.3466 & 0.3781 \\
\hline & & & $(0.5008,0.9729)$ & $(0.4921,0.9025)$ & 0.4720 & 0.4104 \\
\hline & & & $(0.0618,2.5685)$ & $(0.3297,1.8263)$ & 2.5067 & 1.4966 \\
\hline & & \multirow{3}{*}[15]{} & $(0.2618,0.6395)$ & $(0.2505,0.5731)$ & 0.3776 & 0.3226 \\
\hline & & & $(0.4788,0.9783)$ & $(0.4916,0.9183)$ & 0.4994 & 0.4267 \\
\hline & & & $(0.6496,2.8057)$ & $(0.6503,2.1589)$ & 2.1561 & 1.5086 \\
\hline \multirow{9}{*}{50} & \multirow{9}{*}{40} & \multirow{3}{*}[16]{} & $(0.3095,0.6117)$ & $(0.2989,0.5957)$ & 0.3022 & 0.2968 \\
\hline & & & $(0.4879,0.9541)$ & $(0.5116,0.8263)$ & 0.4661 & 0.3147 \\
\hline & & & $(0.0836,2.5993)$ & $(0.3424,1.8137)$ & 2.5157 & 1.4713 \\
\hline & & \multirow{3}{*}[17]{} & $(0.2968,0.6066)$ & $(0.3149,0.6073)$ & 0.3098 & 0.2924 \\
\hline & & & $(0.4553,0.9823)$ & $(0.4654,0.7823)$ & 0.4454 & 0.3169 \\
\hline & & & $(0.1055,2.6416)$ & $(0.2577,1.7017)$ & 2.5360 & 1.4440 \\
\hline & & \multirow{3}{*}[18]{} & $(0.3029,0.6053)$ & $(0.3011,0.5858)$ & 0.3024 & 0.2847 \\
\hline & & & $(0.4754,0.9730)$ & $(0.5081,0.9101)$ & 0.4976 & 0.4020 \\
\hline & & & $(0.0170,2.7283)$ & $(0.3498,1.7981)$ & 2.7112 & 1.4483 \\
\hline
\end{tabular}

$$
\begin{gathered}
\qquad \prod_{i=n_{1}+1}^{m}\left(\psi_{i}(\beta)\right)^{\nu}\left(1+\left(\psi_{i}(\beta)\right)^{\nu}\right)^{\gamma-1} \\
\left.\cdot \exp \left\{-\left(R_{i}+1\right)\left(1+\left(\psi_{i}(\beta)\right)^{\nu}\right)^{\gamma}\right\}\right\},
\end{gathered}
$$

$$
\begin{aligned}
& \pi_{2}^{*}(\beta \mid \gamma, \nu) \propto \beta^{m-n_{1}-1} \\
& \cdot \prod_{i=n_{1}+1}^{m}\left(\psi_{i}(\beta)\right)^{\nu-1}\left(1+\left(\psi_{i}(\beta)\right)^{\nu}\right)^{\gamma-1} \\
& \quad \cdot \exp \left\{-\left(R_{i}+1\right)\left(1+\left(\psi_{i}(\beta)\right)^{\nu}\right)^{\gamma}\right\} .
\end{aligned}
$$

The conditional posterior distributions of $\gamma, \nu$, and $\beta$ in (32), (33) and (34) cannot be reduced analytically to well known distribution, but the plot of them shows that they are similar to normal distribution. So, the Metropolis method is used to generate random samples from this distribution, with normal proposal distribution.

The following algorithm is used to generate $\gamma, \nu$, and $\beta$ from the posterior distribution in the case of IP and then obtain the BEs.
Algorithm 2.

Step 1. Start with $\gamma^{(0)}=\widehat{\gamma}_{\mathrm{MLE}}, v^{(0)}=\widehat{\nu}_{\mathrm{MLE}}$, and $\beta^{(0)}=\widehat{\beta}_{\mathrm{MLE}}$.

Step 2. Set $i=1$.

Step 3. Generate $\gamma^{*}$ from proposal distribution $\mathbf{N}\left(\gamma^{(i-1)}\right.$, $\left.\operatorname{var}\left(\gamma^{(i-1)}\right)\right)$.

Step 4. Calculate the acceptance probability

$$
k\left(\gamma^{(i-1)} \mid \gamma^{*}\right)=\min \left[1, \frac{\pi_{2}^{*}\left(\gamma^{*} \mid \nu^{(i-1)}, \beta^{(i-1)}\right)}{\pi_{2}^{*}\left(\gamma^{(i-1)} \mid \nu^{(i-1)}, \beta^{(i-1)}\right)}\right] .
$$

Step 5. Generate $U \sim U(0,1)$.

Step 6. If $U \leq k\left(\gamma^{(i-1)} \mid \gamma^{*}\right)$, accept the proposal distribution and set $\gamma^{(i)}=\gamma^{*}$. Otherwise, reject the proposal distribution and set $\gamma^{(i)}=\gamma^{(i-1)}$.

Step 7. To generate $\nu^{*}$ do the Steps 2-6 for $\nu$ not $\gamma$.

Step 8. To generate $\beta^{*}$ do the Steps $2-6$ for $\beta$ not $\gamma$.

Step 9. Set $i=i+1$. 
Step 10. Repeat Steps 3-9 N times.

Step 11. Obtain the BEs of $\gamma, \nu$, and $\beta$ using MCMC under SEL function as

$$
\begin{aligned}
& \tilde{\gamma}_{\mathrm{MC}(\mathrm{IP})}=\frac{1}{N-M} \sum_{i=M+1}^{N} \gamma^{(i)}, \\
& \widetilde{\nu}_{\mathrm{MC}(\mathrm{IP})}=\frac{1}{N-M} \sum_{i=M+1}^{N} \gamma^{(i)}, \\
& \widetilde{\beta}_{\mathrm{MC}(\mathrm{IP})}=\frac{1}{N-M} \sum_{i=M+1}^{N} \beta^{(i)} .
\end{aligned}
$$

\section{Interval Estimation}

In this section, the approximate and bootstrap confidence intervals (CIs) of the parameters $\gamma, \nu$, and $\beta$ are derived.

5.1. Approximate Confidence Intervals. In this subsection, the approximate confidence intervals of the parameters are derived based on the asymptotic distributions of the MLEs of the elements of the vector of unknown parameters $\Theta=$ $(\gamma, \nu, \beta)$. It is known that the asymptotic distribution of the MLEs of $\Theta$ is given by Miller [12]:

$$
((\widehat{\gamma}-\gamma),(\widehat{\nu}-\nu),(\widehat{\beta}-\beta)) \sim \mathbf{N}\left(0, \sigma_{i j}\right)
$$

where $\sigma_{i j}, i, j=1,2,3$, is the variance-covariance matrix of the unknown parameters $\Theta=(\gamma, \nu, \beta)$.

The approximate $100(1-\alpha) \%$ two sided confidence intervals for $\gamma, \nu$, and $\beta$ are, respectively, given by

$$
\begin{aligned}
& \left(\widehat{\gamma}_{l}, \widehat{\gamma}_{u}\right)=\widehat{\gamma} \pm Z_{1-\alpha / 2} \sqrt{\sigma_{11}}, \\
& \left(\widehat{v}_{l}, \widehat{v}_{u}\right)=\widehat{v} \pm Z_{1-\alpha / 2} \sqrt{\sigma_{22}}, \\
& \left(\widehat{\beta}_{l}, \widehat{\beta}_{u}\right)=\widehat{\beta} \pm Z_{1-\alpha / 2} \sqrt{\sigma_{33}},
\end{aligned}
$$

where $Z_{q}$ is the 100qth percentile of a standard normal distribution.

5.2. Bootstrap Confidence Intervals. In this subsection, confidence intervals based on the parametric bootstrap method for the unknown parameters $\gamma, \nu$, and $\beta$ using percentile interval are derived; for more details see Efron and Tibshirani [13].

The following algorithm is implemented to obtain a

\begin{tabular}{|c|c|c|c|c|}
\hline$n$ & $m$ & $n_{1}$ & C.S & $\begin{array}{l}\text { Optimal stress } \\
\text { change time }\left(\tau^{*}\right)\end{array}$ \\
\hline \multirow{6}{*}{15} & \multirow{3}{*}{9} & \multirow{3}{*}{3} & {$[1]$} & 3.6359 \\
\hline & & & [2] & 3.7274 \\
\hline & & & {$[3]$} & 3.5404 \\
\hline & \multirow{3}{*}{12} & \multirow{3}{*}{4} & {$[4]$} & 3.4944 \\
\hline & & & {$[5]$} & 3.6542 \\
\hline & & & {$[6]$} & 3.4599 \\
\hline \multirow{6}{*}{25} & \multirow{3}{*}{15} & \multirow{3}{*}{5} & [7] & 3.4273 \\
\hline & & & [8] & 3.5460 \\
\hline & & & [9] & 3.4446 \\
\hline & \multirow{3}{*}{20} & \multirow{3}{*}{7} & [10] & 3.4179 \\
\hline & & & [11] & 3.5405 \\
\hline & & & {$[12]$} & 3.4601 \\
\hline \multirow{6}{*}{50} & \multirow{3}{*}{30} & \multirow{3}{*}{10} & [13] & 2.7102 \\
\hline & & & [14] & 3.5353 \\
\hline & & & [15] & 2.7102 \\
\hline & \multirow{3}{*}{40} & \multirow{3}{*}{15} & [16] & 2.7102 \\
\hline & & & [17] & 3.4954 \\
\hline & & & [18] & 2.7102 \\
\hline
\end{tabular}
bootstrap sample.

\section{Algorithm 3.}

Step 1. From an original data, $\mathbf{y} \equiv\left(y_{1}, \ldots, y_{n_{1}}, y_{n_{1}+1}, \ldots, y_{m}\right)$, compute the MLEs of the parameters $\gamma, \nu$, and $\beta$.
TABLE 7: The optimal change stress time $\tau^{*}$ with true values $\gamma=2$, $\nu=3$, and $\beta=2$.

Step 2. Use $\widehat{\gamma}_{\mathrm{MLE}}, \widehat{v}_{\mathrm{MLE}}$, and $\widehat{\beta}_{\mathrm{MLE}}$ to generate a bootstrap sample $\mathbf{y}^{*}$ with same $R_{i}, i=1,2, \ldots, m$.

Step 3. As in Step 1 based on $\mathbf{y}^{*}$ compute the bootstrap sample estimates $\widehat{\gamma}^{*}, \widehat{\nu}^{*}$, and $\widehat{\beta}^{*}$ of $\widehat{\gamma}_{\mathrm{MLE}}, \widehat{\nu}_{\mathrm{MLE}}$, and $\widehat{\beta}_{\mathrm{MLE}}$, respectively.

Step 4. Repeat Steps 1-3 B times and arrange each estimate in ascending order to obtain the bootstrap sample $\left\{\widehat{\gamma}^{*[1]}, \widehat{\gamma}^{*[2]}, \ldots, \widehat{\gamma}^{*[B]}\right\}, \quad\left\{\widehat{\nu}^{*[1]}, \widehat{\nu}^{*[2]}, \ldots, \widehat{\nu}^{*[B]}\right\}, \quad$ and $\left\{\widehat{\beta}^{*[1]}, \widehat{\beta}^{*[2]}, \ldots, \widehat{\beta}^{*[B]}\right\}$.

Then, the $100(1-\alpha) \%$ percentile bootstrap confidence intervals for $\gamma, \nu$, and $\beta$ are, respectively, given by

$$
\begin{aligned}
\left(\widehat{\gamma}_{l}^{*}, \widehat{\gamma}_{u}^{*}\right) & =\left(\widehat{\gamma}^{*[\alpha B / 2]}, \widehat{\gamma}^{*[(1-\alpha / 2) B]}\right), \\
\left(\widehat{\nu}_{l}^{*}, \widehat{v}_{u}^{*}\right) & =\left(\widehat{\nu}^{*[\alpha B / 2]}, \widehat{v}^{*[(1-\alpha / 2) B]}\right), \\
\left(\widehat{\beta}_{l}^{*}, \widehat{\beta}_{u}^{*}\right) & =\left(\widehat{\beta}^{*[\alpha B / 2]}, \widehat{\beta}^{*[(1-\alpha / 2) B]}\right) .
\end{aligned}
$$

\section{Estimation of Optimal Stress Change Time}

In this section, the optimal change stress time $\tau^{*}$ is found by minimizing the asymptotic variance of MLEs of the model parameters and the acceleration factor. The asymptotic variance of $\widehat{\gamma}, \widehat{\nu}$, and $\widehat{\beta}$ is given by the trace of the inverse of the Fisher information matrix. NMinimize option of Mathematica 7 is used to find the time $\tau^{*}$ which minimize the asymptotic variance of MLEs. Table 7 represents the values of $\tau^{*}$ for different values of $n, m$, and $R_{i}, \quad i=1,2, \ldots, m$. 


\section{Simulation Studies}

In this section, simulation studies are conducted to investigate the performances of the MLEs and BEs in terms of their mean square errors (MSEs) for different choices of $n$, $m$, and $R_{i}, i=1,2, \ldots, m$; the results were concluded in Table 5. Also, the $95 \%$ approximate and percentile bootstrap CIs are computed; the results were concluded in Table 6. The progressive censoring schemes used in the Monte Carlo simulation study are given in Table 1 . The estimation procedure is performed according to the following algorithm.

\section{Algorithm 4.}

Step 1. Specify the values of $n, m, \tau$, and $R_{i}, i=1,2, \ldots, m$.

Step 2. Specify the values of the parameters $\gamma, \nu$, and $\beta$.

Step 3. For given values of the prior parameters $\mu$ and $\lambda$ generate $\nu$ from $\operatorname{Exp}(\lambda)$ and $\gamma$ from $\operatorname{Gamma}(\mu, \nu)$.

Step 4. Use the model given by (6) to generate progressively censored data for given $n, m$; the set of data can be considered as

$$
y_{1 ; m, n}^{R}<\cdots<y_{n_{1} ; m, n}^{R}<\tau<y_{n_{1}+1 ; m, n}^{R}<\cdots<y_{m ; m, n}^{R},
$$

where $R=\left(R_{1}, R_{2}, \ldots, R_{m}\right)$ and $\sum_{i=1}^{m} R_{i}=n-m$.

Step 5. Use the progressive censored data to compute the MLEs of the model parameters. The Newton Raphson method is applied for solving the nonlinear system (11) to obtain the MLEs of the parameters.

Step 6. Compute the BEs of the model parameters relative to SEL function for NIP case and IP case based on Lindley's approximation.

Step 7. Compute the BEs of the model parameters relative to SEL function for NIP case and IP case based on MCMC algorithm, with $N=5500, M=500$.

Step 8. Compute the approximate confidence bounds with confidence levels $95 \%$ for the three parameters of the model.

Step 9. Compute the $95 \%$ bootstrap confidence intervals for the model parameters, using Algorithm 3.

Step 10. Replicate the Steps 3-9, 1000 times.

Step 11. Compute the average values of the mean square errors (MSEs) associated with the MLEs and BEs of the parameters.

Step 12. Do Steps $1-11$ with different values of $n, m$, and $R_{i}, i=$ $1,2, \ldots, m$.

7.1. Illustrative Example. In this subsection, we present an example to illustrate the estimation procedure and the two considered approximate and bootstrap CIs for the parameters $\gamma, \nu$, and $\beta$. In this example, we simulate a sample of size $n=30$, based on population parameter values $\gamma=0.5$, $\nu=0.7$, and $\beta=1.1$. The stress change time $\tau$ is chosen to be equal to 0.9 . Under progressive type-II censoring, $m=20$, $R_{1}=10$, and $R_{i}=0, i=2,3, \ldots, 20$; then the failure times of SSPALT were listed in Table 2. Table 3 presents MSEs of the MLEs and BEs of the model parameters. Table 4 includes 95\% approximate and percentile bootstrap CIs of the model parameters and their lengths.

\section{Conclusion}

In this paper, we considered the statistical inference procedure for the unknown parameters of the $\operatorname{PGW}(\gamma, \nu, 1)$ distribution and the acceleration factor $(\beta)$, when the data are progressive type-II censored from SSPALTs. We obtained the MLEs and BEs of the considered parameters and studied their performance through their MSEs. We also constructed 95\% approximate and bootstrap CIs for the parameters.

From the results in Tables 5-7, we observe the following.

(1) The MSEs of MLEs and BEs of the considered parameters decrease as the sample size increases.

(2) The BEs of the considered parameters obtained from both Lindley's approximation and MCMC method give more accurate results through the MSEs than MLEs.

(3) The BEs of $\gamma, v$ obtained from MCMC method give more accurate results through the MSEs than the BEs obtained from Lindley's approximation.

(4) For the large samples ( $m \geq 20$ ), the BEs of acceleration factor obtained from MCMC method give more accurate results through the MSEs than the BEs of acceleration factor obtained from Lindley's approximation.

(5) For the small samples $(m<20)$, the BEs of acceleration factor obtained from Lindley's approximation give more accurate results through the MSEs than the BEs of acceleration factor obtained from MCMC method.

(6) The bootstrap CIs give more accurate results than the approximate CIs since the lengths of the former are less than the lengths of latter, for different sample sizes, observed failures, and censoring schemes, in most cases.

(7) The optimal stress change time $\tau^{*}$ decreases as the sample size increases.

\section{Conflict of Interests}

The authors declare that there is no conflict of interests regarding the publication of this paper.

\section{Acknowledgments}

The authors would like to thank the two anonymous referees for their constructive comments on an early version of this paper. 


\section{References}

[1] W. Nelson, Accelerated Testing: Statistical Models, Test Plans and Data Analysis, Wiley, New York, NY, USA, 1990.

[2] P. K. Goel, "Some estimation problems in the study of tampered random variables," Tech. Rep. 50, Department of Statistics, Carnegie-Mellon University, Pittspurgh, Pa, USA, 1971.

[3] M. H. DeGroot and P. K. Goel, "Bayesian estimation and optimal designs in partially accelerated life-testing," Naval Research Logistics Quarterly, vol. 26, no. 2, pp. 223-235, 1979.

[4] G. K. Bhattacharyya and Z. Soejoeti, "A tampered failure rate model for step-stress accelerated life test," Communications in Statistics-Theory and Methods, vol. 18, no. 5, pp. 1627-1643, 1989.

[5] D. S. Bai and S. W. Chung, "Optimal design of partially accelerated life tests for the exponential distribution under typeI censoring," IEEE Transactions on Reliability, vol. 41, no. 3, pp. 400-406, 1992.

[6] A. A. Ismail and H. M. Aly, "Optimal planning of failure-step stress partially accelerated life tests under type-II censoring," Journal of Statistical Computation and Simulation, vol. 80, no. 12, pp. 1335-1348, 2010.

[7] M. M. Abdel-Ghani, "The estimation problem of the log-logistic parameters instep partially accelerated life tests using type-I censored data," The National Review of Social Sciences, vol. 41, no. 2, pp. 1-19, 2004.

[8] N. Balakrishnan and R. Aggarwala, Progressive Censoring: Theory, Methods, and Applications, Birkhäuser, Boston, Mass, USA, 2000.

[9] V. Bagdonavičius and M. Nikulin, Accelerated Life Models: Modeling and Statistical Analysis, Chapman \& Hall/CRC, 2002.

[10] D. V. Lindley, "Approximate Bayes methods," Trabajos de Estatadistica, vol. 31, pp. 223-237, 1980.

[11] N. Metropolis, A. W. Rosenbluth, M. N. Rosenbluth, A. H. Teller, and E. Teller, "Equation of state calculations by fast computing machines," The Journal of Chemical Physics, vol. 21, no. 6, pp. 1087-1092, 1953.

[12] R. G. Miller, Survival Analysis, Wiley, New York, NY, USA, 1981.

[13] B. Efron and R. J. Tibshirani, An Introduction to the Bootstrap, vol. 57 of Monographs on Statistics and Applied Probability, Chapman and Hall, London, UK, 1993. 


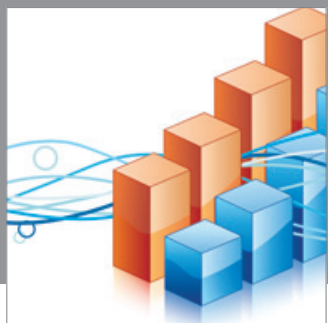

Advances in

Operations Research

mansans

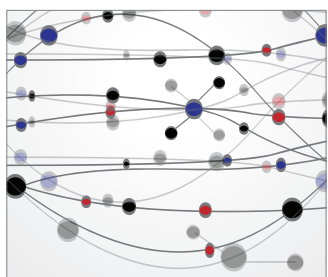

The Scientific World Journal
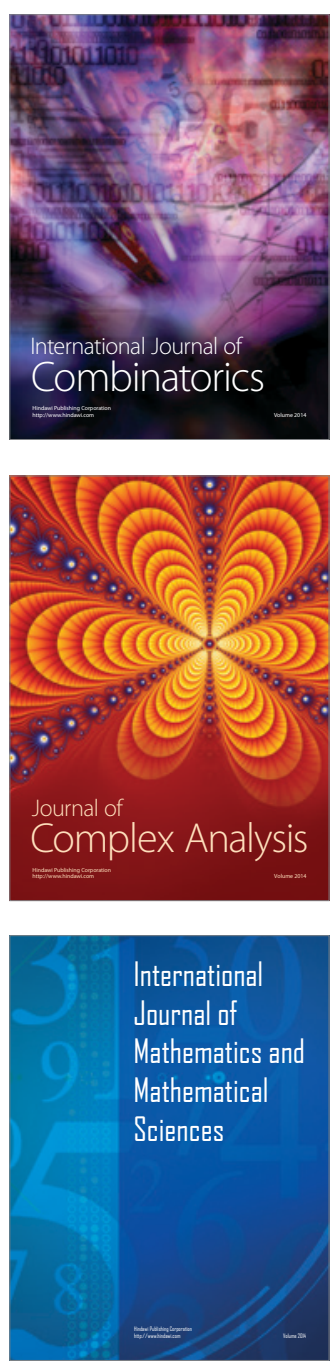
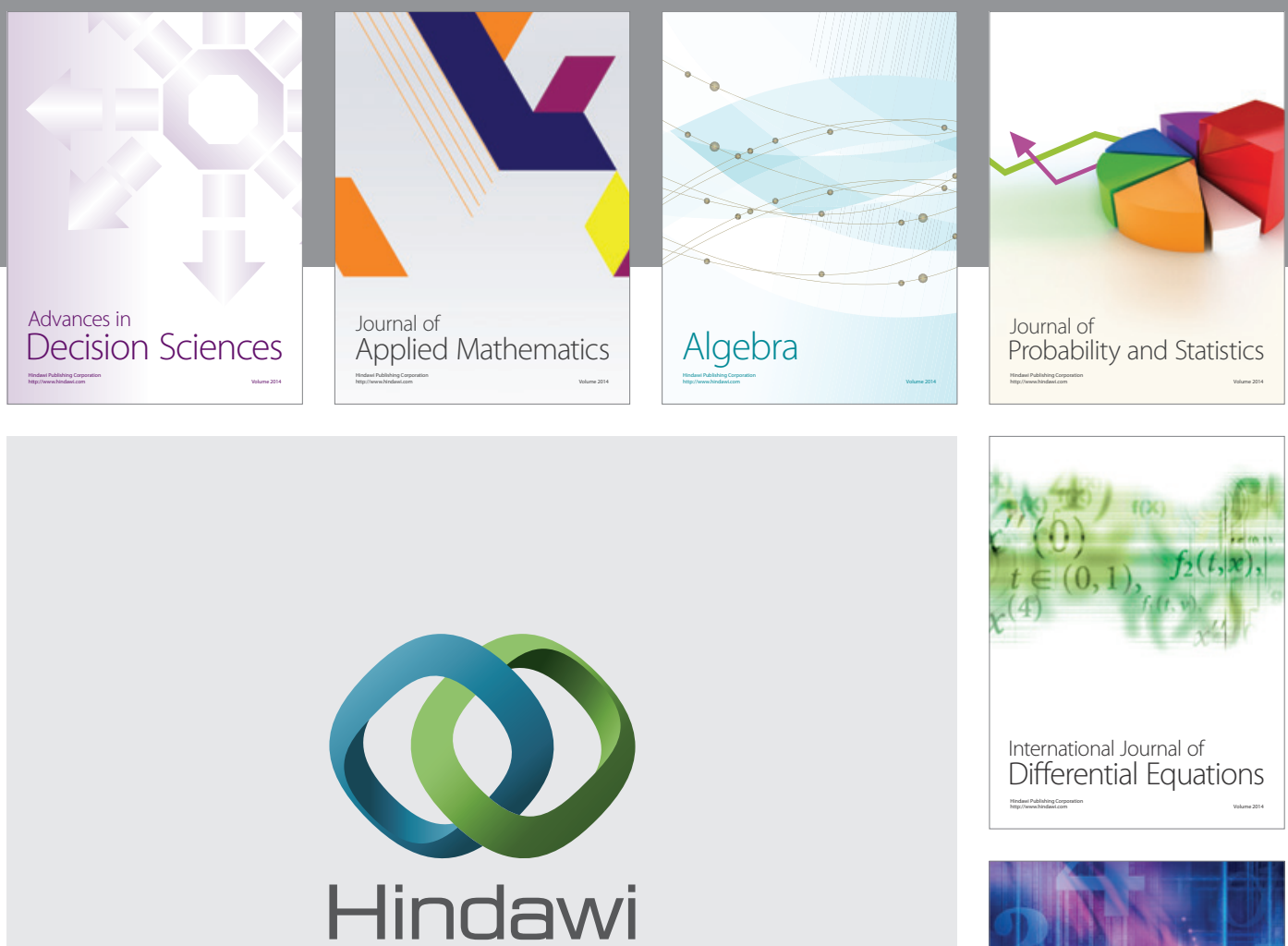

Submit your manuscripts at http://www.hindawi.com
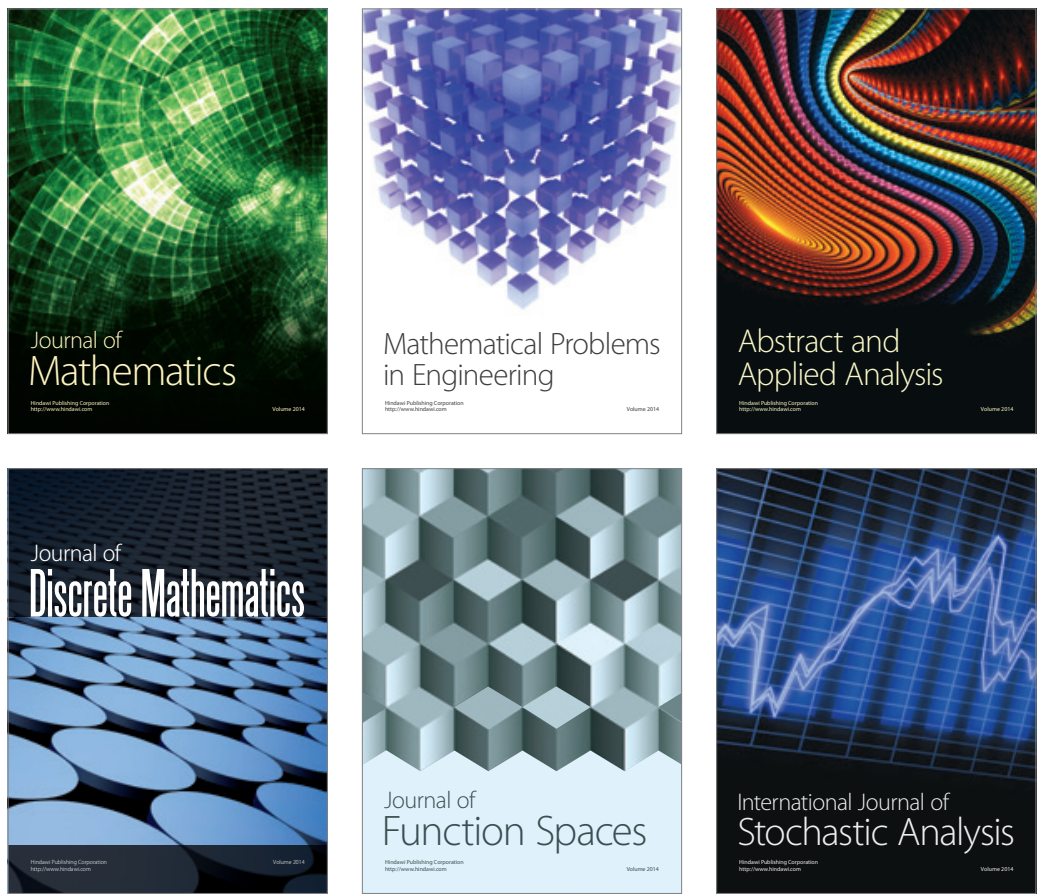

Journal of

Function Spaces

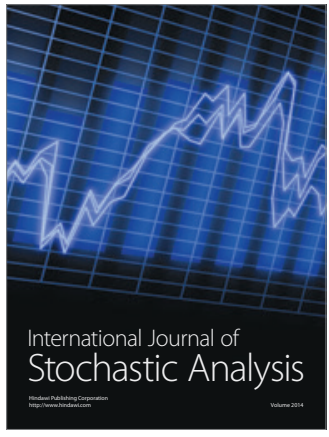

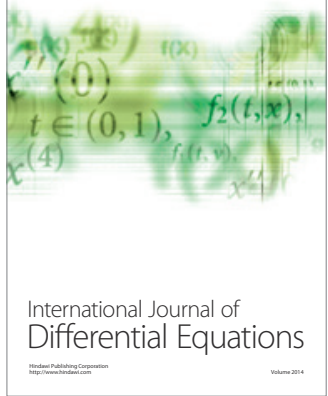
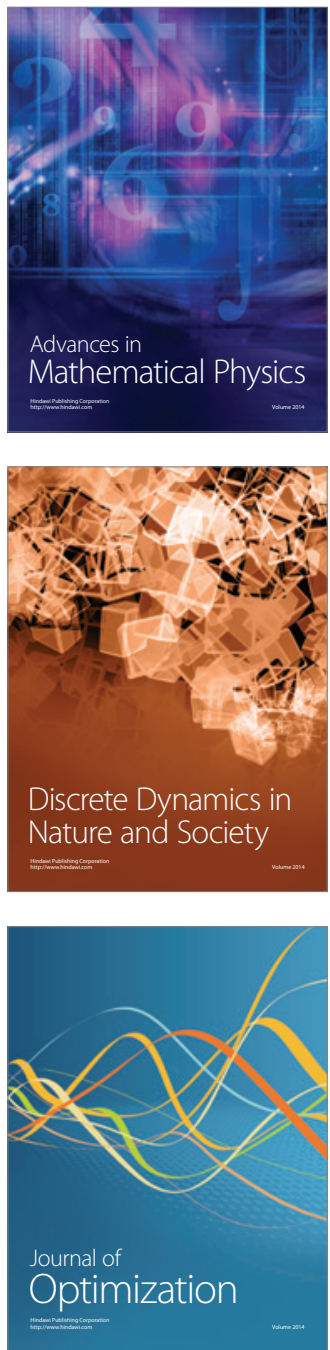